\title{
Aplicando o Sloodle como um Ambiente de Ensino e Aprendizagem
}

\author{
Adriana D. Dantas ${ }^{1,2}$, Lucilene Maciel $^{1}$, David Lima ${ }^{1,2}$, Thais Castro ${ }^{1}$ \\ Isomar Lima ${ }^{1}$, Antonio R. Neto ${ }^{2}$ \\ ${ }^{1}$ Instituto de Computação - Universidade Federal do Amazonas (UFAM) \\ Manaus, Amazonas \\ ${ }^{2}$ Instituto Federal de Educação, Ciência e Tecnologia do Amazonas (IFAM) \\ Manaus, Amazonas \\ \{david.lima, thais\}@icomp.ufam.edu.br, dricadoroteu@gmail.com
}

\begin{abstract}
This article presents a case study conducted at a university that uses Sloodle what is a virtual environment for teaching and learning in three dimensions, Sloodle derives from the junction of two virtual environments, Moodle and Second Life. In order to involve the student and insert him in a educational environment one good interaction becomes a great ally for help in accomplishing this purpose. The objective of this case study is to evaluate the interaction and usability of Sloodle. The results contribute to improve the usability of this platform by identifying the advantages and disadvantages, as well as contributing in new research Virtual Environments for Teaching in three dimensions.
\end{abstract}

Resumo. Este artigo apresenta um estudo de caso realizado em uma universidade que utiliza o Sloodle que é um ambiente virtual de ensino e aprendizagem em três dimensões, o Sloodle deriva da junção de dois ambientes virtuais, o Moodle e o Second Life. No intuito de envolver o aluno e inseri-lo no ambiente educacional, uma boa interação torna-se uma grande aliada para contribuir na realização dessa finalidade. O objetivo desse estudo de caso é avaliar a interação e usabilidade do Sloodle. Os resultados obtidos contribuem para melhorar a usabilidade desta plataforma através da identificação das vantagens e desvantagens, além de contribuir para novas pesquisas em Ambientes Virtuais de Ensino em três dimensões.

\section{Introdução}

A falta de tempo é uma problemática crescente entre vários indivíduos, e a necessidade de obter conhecimento para ser absolvido pelo mercado de trabalho tão competitivo se torna imprescindível na vida das pessoas.

Consoante com essa problemática, a educação à distância $(\mathrm{EaD})$ surgiu como uma possibilidade de levar educação onde quer o aluno esteja, dando a ele a oportunidade de gerenciar o seu tempo [TORI, 2010].

Uma tecnologia bastante utilizada na EaD é o uso de Ambientes Virtuais de Aprendizagem (AVA). O AVA é um exemplo de Tecnologia da Informação e Comunicação (TIC), que funciona como uma plataforma que dispõe de um ambiente com aparatos pedagógicos e educacionais que são integrados a práticas de ensino que 
são disponibilizados por professores que interagem com os alunos de forma prática [DELWICHE, 2006].

Segundo Douguimas (2009), várias empresas e instituições de ensino já utilizam a $\mathrm{EaD}$, trazendo vários benefícios, tanto para os alunos e professores que não precisam está presencialmente na sala, como para a Instituição que não precisa dispor de um local físico para alocar os alunos.

As TICs devem então dar suporte a mediação e prover um ambiente onde estudantes e professores podem estabelecer uma presença social. Um exemplo das TICs utilizadas na EaD é o ambiente Sloodle que tem o objetivo de aumentar e melhorar a interação, comunicabilidade e aprendizagem dos alunos em um ambiente tridimensional onde eles serão os principais personagens de um jogo de aprendizagem da vida real [HODGE et al. 2010].

A principal contribuição deste artigo é identificar as vantagens e desvantagens do ambiente no intuito de melhorar a interação do usuário com a plataforma através de um relato de experiência no ambiente de ensino à distância Sloodle.

\section{Mundos Virtuais de Aprendizagem}

Ambientes Virtuais Colaborativos (Collaborative Virtual Environments - CVE) são considerados espaços onde ocorrem simulações de mundos reais ou imaginários, sendo formados pelo próprio espaço virtual, através do qual seus participantes podem interagir, compartilhar informacões, colaborar no desenvolvimento de atividades e fazer uso de artefatos disponíveis neste ambiente [BAINBRIDGE, 2010].

De acordo com Bainbridge (2010), CVEs se popularizaram com a pespectiva de sua representação através de três dimensões (3D), onde usuários podem potencializar sua participação no espaço através da personificação de um avatar, sendo possível criar uma identidade mais próxima do mundo real.

No contexto deste artigo, os mundos virtuais podem ser denominados como ambientes virtuais $3 \mathrm{D}$ onde as pessoas podem interagir de maneira comparável ao mundo real, seja para o trabalho ou para o lazer. Os mundos virtuais combinam gráficos 3D interativos, tecnologia de simulação, realidade virtual e mídia digital, fornecendo aos usuários, habilidades para se comunicar, colaborar e explorar HODGE et al. 2009; RYMASZEWSKI 2007).

Esse conjunto de tecnologias fornece uma interface para um mundo tridimensional, de modo que o usuário acredite estar realmente neste mundo e, intuitivamente, comece a interagir com esse ambiente imersivo e dinâmico [MATTAR, 2008].

\section{Moodle e Second Life}

O Moodle (Modular Object-Oriented Dynamic Learning Environment) foi criado em 1999, cuja base pedagógica é a abordagem social-construcionista da educação [SALVIN, 2010].

O Moodle, como plataforma de gestão do ensino/aprendizagem, facilita a interação professor-aluno, bem como a apresentação, entrega e correção de trabalhos em ambiente de sala de aula virtual (disponível em qualquer momento, e potencialmente em 
qualquer local com acesso a internet) [LEGOINHA et al. 2006].

De acordo com Wagner et al. (2012) o Second Life teve sua primeira versão lançada em 2003, com apenas dezesseis servidores e mil usuários. Hoje, possui mais de três mil servidores e aproximadamente cinco milhões de usuários registrados.

Segundo Valente et al. (2007), as experiências pedagógicas que têm sido realizadas em mundos virtuais, mais especificamente no Second Life, chamam a atenção para a importância do "espaço de aprendizagem". O grau de envolvimento e imersão dos alunos com o conteúdo dos cursos, os colegas e o próprio professor, em um ambiente de realidade virtual 3D como o Second Life, não parece ser facilmente reproduzível nos ambientes de aprendizagem tradicionais, como Blackboard, Teleduc e Moodle.

\section{Sloodle}

De acordo com Wagner et al. (2012), o Sloodle é um sistema que integra mundos virtuais 3D desenvolvidos e integrados ao Second Life e atualmente também ao OpenSim, com o sistema de gerenciamento de aprendizagem de código aberto Moodle. Isto possibilita vantagens como a grande demanda por aprendizagem através de ambientes imersivos.

Na prática, o Sloodle é um jogo do Second Life em uma região/mapa de sala de aula, sendo este mapa vinculado a uma disciplina no Moodle, o que gera toda a documentação da aula. O Sloodle adiciona ao Second Life objetos existentes em uma sala de aula real como cadeiras, quadros, salas entre outros. Gera também interações existentes em um ambiente de aprendizagem presencial como: quiz, provas, perguntas e respostas, o avatar levantando o dedo para tirar dúvidas dentre outras formas de interação [WANKEL, 2009].

\section{Trabalhos Correlatos}

Os trabalhos relatados levam em consideração a importância da interação do usuário final, ou seja, o discente. Nesse sentido, Frozza et al. (2009), afirma que agentes virtuais que expressam emoções vêm sendo desenvolvidos com o intuito de promover uma maior sensação de proximidade entre o usuário e o sistema.

A importância da aparência visual como um agente pedagógico motivador para os alunos é relatada no artigo de Odakura et al.(2012). Nesse sentido, Azevedo e Elia (2011) apresentam um plano pedagógico que faz uso do software Open Simulator para modelar o ambiente imersivo em três dimensões que visa resgatar o interesse do alunado pelo ensino, a partir da adaptação de conteúdos pedagógicos ao ambiente virtual.

De acordo com Amaral et al. (2012) apresenta todo o processo de implementação de um laboratório virtual de geometria dentro do metaverso do Opensim, esta investigação tem o objetivo de mostrar o domínio sobre a ferramenta que deve proporcionar maior autoria aos usuários do LV.

Em seu artigo Voss et al. (2013) apresenta um estudo de caso qualitativo sobre ambientes em três dimensões para dispositivos móveis. O musert é um museu virtual que apresenta como um diferencial a recomendação personalizada de conteúdo a partir de sua plataforma em 3D é mostrado por Lima et al. (2012), como uma ferramenta educacional eficiente, pois disponibiliza informações aos seus visitantes de forma 
simples e de fácil compreensão.

No trabalho de Cazzeta et al. (2012) é apresentado o Kinble, um ambiente colaborativo centrado em um jogo $3 \mathrm{~d}$, onde o aluno é apresentado a um ambiente competitivo, é estimulado a responder as questões para conseguir uma melhor posição no ranking de pontuação. A sua arquitetura destaca-se por ter um módulo de cadastro de questões, onde um professor de qualquer disciplina de nível médio pode alimentá-lo e compartilhar informações com outros professores. Já Rossito et al. (2012) mostra em seu artigo uma metodologia que seja aplicável a todos os softwares $3 \mathrm{~d}$ interativos (S3DI) com foco educacional. Esta metodologia baseia-se no estabelecimento de uma taxonomia.

As discussões sobre a importância do ensino e aprendizagem através dos ambientes virtuais em três dimensões devem ser mais exploradas, pois no âmbito educacional essas ferramentas vêm oferecendo maiores níveis de interatividade entre os discentes.

\section{Estudo de Caso}

A capacidade heurística é uma contribuição do estudo de caso, pois pode levar a descobrir novos sentidos, expandir suas experiências ou confirmar o que já se sabia. O estudo de caso tem um potencial enorme de contribuição aos problemas da prática educacional, ao fornecer informações valiosas que permitem também decisões políticas [YIN, 2005].

Estudo de caso é uma investigação empírica que abrange planejamento, técnicas de coleta de dados e análise dos mesmos [André, 2005a]. Neste contexto, André (2005b) completa afirmando que estudo de caso é a possibilidade de fornecer uma visão profunda, ampla e integrada de uma unidade social complexa. Ao retratar situações reais sem prejuízo de sua dinamicidade natural, o estudo de caso se configura vantajoso.

\section{Aplicando o Estudo de Caso no Sloodle}

O objetivo da avaliação é tentar melhorar o ambiente, através da identificação de suas vantagens e desvantagens, uma vez que a imersão do ambiente trás para o aluno uma forma diferente de interação e comunicação. A contribuição para o ensino à distância é chamar a atenção do aluno tornado o ambiente interessante, para tentar diminuir assim o índice de evasão dos cursos à distância.

\subsection{Preparação do Ambiente}

Para que a avaliação fosse realizada, vários passos foram seguidos, como a preparação do ambiente com a instalação do Sloodle. Além desta etapa, foram introduzidos os principais recursos para desenvolvimento de mundos virtuais utilizando estas ferramentas. Foram realizadas três etapas para instalação do Sloodle:

1. Instalação das ferramentas Moodle, SecondLife e OpenSim.

2. Instalação de recursos do Sloodle no Moodle e no OpenSim.

3. Construção do ambiente virtual no OpenSim.

O OpenSim foi utilizado por ser uma plataforma de código livre que permite a criação e edição de ambientes em 3D e pode ser executado em dois modos, StandAlone e Grid. O OpenSim foi utilizado para importação de ambientes disponibilizados 
gratuitamente (denominados ilhas) e os dois modos de execução foram testados.

É possível registrar atividades do Second Life no Moodle e importar recursos do Moodle para o Second Life. Através do OpenSim, importaram-se ambientes contendo prédios e auditórios, em formato oar (formato para importar ilhas inteiras) ou no formato iar (formato para objetos desde cadeiras a prédios).

Para a modelagem virtual da ilha, foram utilizados os seguintes recursos criados pelo Sloodle no Moodle: Controller, Choice Vertical, Choice Horizontal, Primdrop, Quizpile, Lite Toolbar e Login Zone.

O Controller é um objeto do ambiente virtual 3D que faz conexão da disciplina do Moodle com a região da disciplina na ilha do Second Life. Este elemento é responsável por criar e controlar os demais objetos na ilha, tais como: o Choise Vertical, Choise Horizontal e Primdrop.

O Choice Vertical e Choise Horizontal são objetos do ambiente virtual 3D que representa o Quiz, recurso que simula uma pesquisa de opinião sobre determinado assunto do Moodle no Second Life.

\subsection{Cenários do Estudo de Caso}

Foram apresentados aos alunos dois ambientes distintos para cada atividade: um para atividade individual, outro para reunião. $\mathrm{O}$ ambiente para atividade individual foi uma sala de aula convencional, onde os alunos podiam ver as atividades propostas, como mostra a Figura 1.

O segundo ambiente apresentado aos alunos foi uma sala de reuniões onde um pequeno grupo se reuniu para realizar uma atividade do problema de raciocínio lógico.

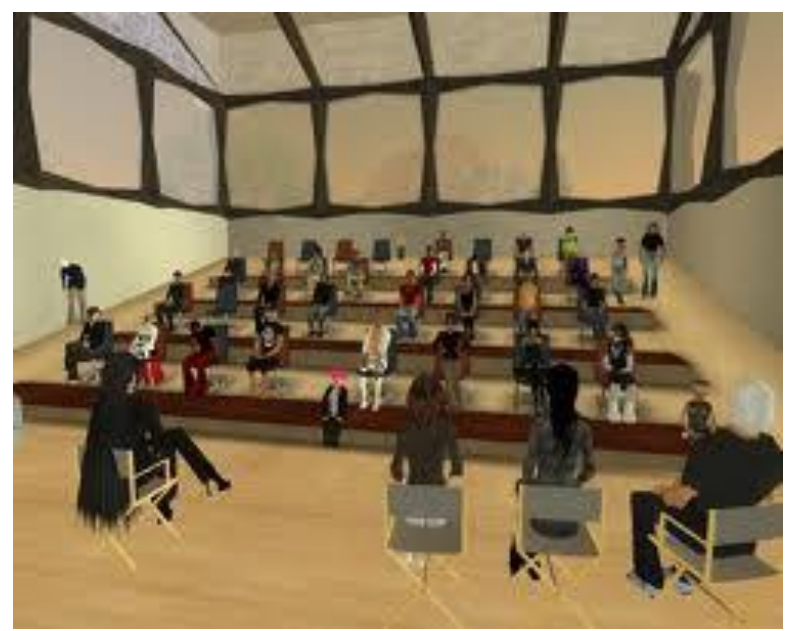

Figura 1. Sala de aula no ambiente Sloodle.

\subsection{Perfil dos Participantes}

Os participantes são alunos do Campus Manaus Centro do Instituto Federal do Amazonas, que cursam a disciplina Introdução à Informática no Curso de graduação em Engenharia Civil. A turma é formada por 30 alunos com idade entre 18 a 39 anos e sem nenhuma experiência na utilização do ambiente de aprendizagem Sloodle. 


\subsection{Execução da Avaliação}

O estudo de caso foi realizado em abril de 2014, no laboratório de informática, onde cada participante estava em uma máquina e não poderia ter nenhum tipo de comunicação no ambiente real, apenas no virtual.

Foram formados seis grupos de cinco pessoas para a conclusão das atividades, onde primeiro foram realizadas as atividades em grupo e posteriormente as atividades individuais para que fossem analisadas. Vale ressaltar que antes dos testes os participantes assinaram um Termo de Consentimento Livre e Esclarecido que descrevia o processo da pesquisa, o objetivo e a importância da veracidade da resposta de cada participante para a pesquisa.

Durante o processo de avaliação os usuários foram instruídos a realizar algumas atividades específicas de uma sala virtual comum ao Moodle, porém em um ambiente 3D utilizando o Sloodle. No próprio ambiente os alunos pediam instruções das atividades que eles sentiam dificuldades em realizar. Isso ficava por conta de um avatar na sala de aula.

O processo de avaliação durou aproximadamente 3 horas e cinquenta minutos para todas as equipes, todas elas realizaram as atividades simultaneamente. As atividades foram divididas em duas fases: atividades individuais e atividades em grupo.

\subsubsection{Atividades Individuais}

Os alunos acessaram o ambiente e realizaram as atividades que estavam expostas para eles, sendo:

1. Responder a um questionário onde as respostas estavam em um texto disponibilizado na sala onde eles deveriam fazer o download do arquivo para responder as perguntas.

2. Fazer o upload do arquivo com as respostas.

3. Responder no fórum a respeito do texto lido.

4. Enviar uma mensagem dizendo que terminou a avaliação.

\subsubsection{Atividades em Grupo}

Os alunos foram divididos em grupos obedecendo a ordem alfabética, sendo:

1. Resolver um problema de raciocínio lógico entre os membros do grupo. Para cada grupo foi distribuído uma dica diferente no intuito de ajudá-los a resolver o problema.

2. Para resolver o problema, os membros do grupo deveriam interagir usando o chat como meio de comunicação.

3. Enviar uma mensagem dizendo que terminou a avaliação.

O Estudo de caso foi conduzido com a turma do $1^{\circ}$ período do curso, pois assim a pesquisa se tornou mais homogênea com perfis diferenciados de conhecimento em informática, atingindo o público-alvo: os novos jovens com acesso ao ensino superior. A pesquisa contou com o total de trinta alunos, dois professores e um tutor, dos quais somente vinte alunos alunos participaram e responderam todas as atividades propostas.

Os resultados foram extraídos empíricamente sob a observação do pesquisador, de forma quantitativa e qualitativa através de questionários utilizando perguntas do tipo 
objetivas e discursivas.

\section{Descrição das Questões do Estudo de Caso}

A proposta do trabalho é de avaliar o funcionamento e aplicabilidade do Sloodle, a satisfação e o interesse dos discentes por esta ferramenta, a partir desta proposta o pesquisador elaborou as seguintes questões e coletou as respostas para o desenvolvimento deste artigo.

No início da proposta, os alunos demonstraram muita excitação com a notícia de utilizar um jogo para realizar a interação na disciplina. Depois de certa dificuldade os alunos passaram a interagir e a pedir o Sloodle para utilizar com maior frequência após a ótima experiência vivida com a ferramenta. As questões do estudo de caso são:

a) Com relação a usabilidade do sloodle você achou?

Sloodle?

b) Como foi o seu interesse com relação ao estudo da disciplina através do Sloodle?

c) Como foi a interação entre você e seus colegas de classe na utilização do

d) Como você se sente em relação a utilização da plataforma?

e) O que você achou em relação ao ambiente da plataforma em geral?

Para cada pergunta mencionada acima foi gerada uma tabela, na qual mostra os resultados da pesquisa de forma mais clara.

Tabela 1. Com relação a usabilidade do Sloodle você achou?

\begin{tabular}{|c|c|c|c|c|c|}
\hline Nível de Avaliação & $\begin{array}{c}\text { Muito } \\
\text { Difícil }\end{array}$ & Difícil & $\begin{array}{c}\text { Mais ou } \\
\text { Menos }\end{array}$ & Fácil & $\begin{array}{c}\text { Muito } \\
\text { Fácil }\end{array}$ \\
\hline Quantidade de Alunos & 2 & 2 & 4 & 10 & 2 \\
\hline
\end{tabular}

Embora algumas dificuldades tenham sido encontradas no momento do estudo de caso, o seu uso não foi prejudicado como mostra a tabela acima, onde $80 \%$ dos discentes demonstraram ter um grau de fácil utilização com relação a usabilidade do sistema.

Tabela 2. Como foi o seu interesse com relação ao estudo da disciplina através do Sloodle?

\begin{tabular}{|c|c|c|c|c|c|}
\hline Nível de Avaliação & $\begin{array}{c}\text { Sem } \\
\text { Interesse }\end{array}$ & $\begin{array}{c}\text { Pouco } \\
\text { Interessado }\end{array}$ & $\begin{array}{c}\text { Meio } \\
\text { Interessado }\end{array}$ & Interessado & $\begin{array}{c}\text { Muito } \\
\text { Interessado }\end{array}$ \\
\hline Quantidade de Alunos & 0 & 0 & 2 & 7 & 11 \\
\hline
\end{tabular}

Um fator relevante para a pesquisa foi perceber o quanto os alunos estavam ansiosos para manipular a plataforma, e através dessa reação foram formuladas algumas perguntas bem específicas conforme mostra a tabela 2 acima, onde $90 \%$ dos alunos confirmaram que estavam interessados ou muito interessados pela ferramenta.

Tabela 3. Como foi a interação entre você e seus colegas de classe na utilização do Sloodle?

\begin{tabular}{|c|c|c|c|c|c|}
\hline Nível de Avaliação & Péssimo & Ruim & Boa & Ótima & Excelente \\
\hline Quantidade de Alunos & 0 & 1 & 4 & 7 & 8 \\
\hline
\end{tabular}


A interação entre os alunos é outro item importante que foi avaliado durante a pesquisa. O estudo de caso comprovou que mais de $90 \%$ dos alunos definiram que a interação foi muito maior quando se utilizou o Sloodle, enquanto só $0,1 \%$ relataram que tiveram pouca interação com outros usuários, conforme a tabela 3.

Tabela 4. Como você se sente em relação a utilização da plataforma?

\begin{tabular}{|c|c|c|c|c|c|}
\hline Nível de Avaliação & $\begin{array}{c}\text { Muito } \\
\text { Insatisfeito }\end{array}$ & Insatisfeito & $\begin{array}{c}\text { Meio } \\
\text { Satisfeito }\end{array}$ & Satisfeito & $\begin{array}{c}\text { Muito } \\
\text { Satisfeito }\end{array}$ \\
\hline Quantidade de Alunos & 0 & 2 & 3 & 6 & 9 \\
\hline
\end{tabular}

Notou-se através da observação do docente, a existência de um pequeno grupo nomeado como "leitores" que apesar de estarem incluídos no processo não realizaram a interação da forma prevista e não responderam a todas as atividades.

Com relação a satisfação dos alunos ao usar a plataforma foi percebido um grande prazer por parte dos discentes na exploração dos recursos tecnológicos do sistema e na interação virtual como mostra a tabela 4 acima.

Tabela 5. 0 que você achou em relação ao ambiente da plataforma em geral?

\begin{tabular}{|c|c|c|c|}
\hline Nível de Avaliação & $\begin{array}{c}\text { Falta } \\
\text { Melhorar }\end{array}$ & $\begin{array}{c}\text { Está } \\
\text { Bom }\end{array}$ & $\begin{array}{c}\text { Está } \\
\text { Excelente }\end{array}$ \\
\hline Quantidade de Alunos & 4 & 7 & 9 \\
\hline
\end{tabular}

Embora tenha sido positiva a pesquisa com relação ao ambiente da plataforma em geral, como mostra a tabela 5, é importante que sejam feitas novas pesquisas, avaliações e experimentos da plataforma em grande escala, para que sejam apontadas as vantagens e desvantagens da ferramenta, e que posteriormente elas possam ser aperfeiçoadas. Dentre a pesquisa qualitativa realizada, podemos destacar os seguintes pontos:

- A interação entre os sujeitos envolvidos ultrapassou os limites das salas de aulas, aproximou os indivíduos e aumentou a transmissão do saber.

- A interação se deu de forma dialogada e não hierarquizada.

- A experiência provocou tanto nos professores quanto nos estudantes uma reflexão sobre o processo de ensino-aprendizagem.

- Os discentes demonstraram excitação com o uso de tecnologias e se mostraram mais interessados em progredir no Curso de Ciência da Computação para descobrir mais sobre a tecnologia.

- Alguns dos discentes indicaram algumas dificuldades com o processo, mas não temeram pela tecnologia e enfrentaram as barreiras.

- O contexto colaborativo gerou maior inclusão social e relacionamentos dentro da classe de alunos.

\section{Análise dos Resultados}

O Sloodle se apresentou como uma ferramenta promissora para a interação dos discentes, despertando o interesse dos alunos quanto ao aprendizado e fazendo o que se propõe: adaptar-se à realidade deste novo perfil do aluno cada vez mais exigente com relação a usabilidade da ferramenta. 
Os mundos de três dimensões como o Second Life podem ser considerados como redes sociais em três dimensões onde as pessoas podem criar comunidades colaborativas, criar e editar objetos como uma wiki tridimensional. De acordo com Savin (2010), o Sloodle realiza essa interatividade com o Moodle visando promover a educação.

O Estudo de caso nesta ferramenta com os discentes só fortalecem o dito pelos autores, é preciso experimentar o mundo de três dimensões e saber utiliza-lo. A ferramenta é uma tecnologia e não um rival. Apesar de toda a qualidade encontrada com o Sloodle e o processo de ensino aprendizado através dele, há de se considerar as configurações mínimas exigidas tanto a nível de hardware, acesso à internet e características físicas, quanto a nível de conhecimento prévio, facilidade de interação com computador entre outros fatores.

Conforme Legoinha (2006) em cinco anos, a Internet 3D será tão importante para o trabalho quanto a web é hoje. Profissionais de gestão da informação e do conhecimento devem começar a investigar e experimentar com mundos virtuais.

\section{Trabalhos Futuros}

Para trabalhos futuros, verificou-se que é necessário fazer mais avaliações de ambientes 3D no intuito de melhorar as ferramentas e os métodos de avaliação de interação e comunicabilidade já existentes. Fazer pesquisa utilizando os logs dos usuários, com esse recurso as informações se tornam precisas, fazer mais experimentos com poucas pessoas e com mais atividades para os alunos sentirem a comunicação do ambiente. Talvez fosse importante também avaliar o nível de satisfação da utilização do Sloodle como apoio a EaD.

$\mathrm{Na}$ discussão das atividades em grupo no item 2, foram levantados alguns questionamentos sobre o comportamento dos grupos, essas observações foram descritas a partir dos vídeos e olhar do avaliador e não do aluno, para trabalhos futuros seria importante fazer um estudo de caso com mais questões, dessa forma o trabalho ficaria mais completo com a visão real do participante de forma mais diversificada das vantagens e desvantagens do ambiente avaliado.

Há necessidade de fazer mais avaliações com outras ferramentas que também utilizam os ambientes tridimensionais para comunicação e colaboração de atividades ou ainda utilizar versões mais novas da mesma ferramenta, vale ressaltar que o Moodle instalado para os testes dessa pesquisa foi a versão 2.6 e que uma nova versão do ambiente foi lançada recentemente.

\section{Referências}

Amaral,É.; Avila, B. G.; Tarouco, M. R. L. (2012) Aspectos teóricos e práticos da implantação de um laboratório virtual no OpenSim. In: Anais do $23^{\circ}$ Simpósio Brasileiro de Informática na Educação, Rio de Janeiro.

Andre, M. E. D. A. (2005a) Etnografia da prática escolar. Papirus Editora.

Andre, M. E. D. A. (2005b) Estudo de Caso em Pesquisa e avaliação educacional. Brasília: Liber Livro Editora.

Azevedo, C.E.; Elia, M.F. (2011) Proposta de uma Aplicação de Mundos Virtuais na Educação usando o Open Simulator com diferentes requisitos tecnológicos. In: Anais do XXII SBIE - XVII WIE Aracaju. 
Bainbridge, W. S. (2010) Online worlds: convergence of the real and the virtual. London: Springer,.

Delwiche, A. (2006) Massively multiplayer online games (MMOs) in the new media classroom. J. Educational Technology \& Society 9, 3, 160-172.

Douguimas, M. (1999) Reading and Writing for Internet Teaching.

Cazetta P.P.; Machado F. V.; Santos, P. C.; Figueiredo A. M. O.; Sant'ana L. S.; Junior, N. A. S.; Dutra; S. F.; Clua E. (2011) Uma Proposta de Jogo Educacional 3D com Questões Didáticas. In Anais do XXII SBIE - XVII WIE Aracaju.

Frozza, R.; Silva, A. K.; Lux, B.; Cruz, M. E. J. K.; Borin, M. (2009) Proposta de uma Aplicação de Mundos Virtuais na Educação usando o Open Simulator com diferentes requisitos tecnológicos. In: Simpósio Brasileiro de Informática na Educação. n 20, 2009, Florianópolis. Anais, Florianópolis, p. 1-10.

Hodge, E., Collins, S. e Giordano, T. (2011) The virtual worldsh: how to use Second Life and other 3D virtual environments. Sudbury: Jones and Bartlett.

Legoinha, P., Pais, J. e Fernandes, J. (2006) O Moodle e as comunidades virtuais de aprendizagem. VII Congresso Nacional de Geologia de Portugal, 1-4.

Lima, J. D.; de Moura, I.B.; Neto, F.M.M.; Maia, P.S.S. (2012) Musert: Um Museu Virtual em 3D com Recomendação Personalizada de Conteúdo. In: Anais do $23^{\circ}$ Simpósio Brasileiro de Informática na Educação, Rio de Janeiro.

Mattar, J. (2008) O uso do Second Life como ambiente virtual de aprendizagem. Fonte, Belo Horizonte, ano, 5, 88-95.

Odakura V.; Silva J.H. ; Couto, L. S. (2012) Aparência de um agente pedagógico animado para um ambiente virtual de aprendizagem. In: Anais do $23^{\circ}$ Simpósio Brasileiro de Informática na Educação, Rio de Janeiro.

Palloff, R. M. e Pratt K. (2002) Construindo Comunidades de Aprendizagem no Ciberespaço. Porto Alegre: Artmed.

Raposo, A. B. (2011) Ambientes Virtuais Colaborativos. In: Sistemas Colaborativos (Org. Pimentel, M; Fuks, H). Rio de Janeiro: Elsevier, 94-109.

Rymaszewski, M. (2007) Second Life: The Official Guider. New Jersey: Wiley publishing.

Savin-Baden, M. A. (2010) Practical guide to using Second Life in higher education. New York: McGraw-Hill.

Sloodle About It. Disponível em $<\underline{\text { http: } / / w w w . s l o o d l e . o r g / b l o g} /$ ?page $\mathrm{id}=2>$. Acesso em abril de 2014.

Tori, R. (2010) Educação sem distância: as tecnologias interativas na redução de distâncias em ensino e aprendizagem. São Paulo: Senac.

Valente, C. e Mattar, J. (2007) Second Life e web 2.0 na educação: o potencial revolucionário das novas tecnologias. São Paulo: Novatec.

Wagner, R., Piovesan, S. D. e Santarosa, L. (2012) Uma proposta de integração do metaverso Virtualtchê ao sloodle. Nuevas Ideas en Informática Educativa, TISE.

Wankel, C. e Kingsley, J. (2009) Higher education in virtual worlds. Bingley: Emerald.

Yin, R.K. (2005) Estudo de caso: planejamento e métodos. 3. ed. Porto Alegre: Bookm. 